\title{
P-64
}

\section{Preparation and Characterization of Solid Dispersions of Orthosiphon Staminues Ethanolic Extract in PVP and Poloxamer 407}

\author{
Noor Hafizoh Saidan*, Abdalrahim A.F. Aisha and Zhari Ismail \\ Department of Pharmaceutical Chemistry, School of Pharmaceutical Sciences, Universiti Sains Malaysia (USM), 11800 \\ Minden, Pulau Pinang, Malaysia; E-mail: zhari@usm.my
}

Orthosiphon stamineus (Lamiaceae) or Misai kucing has been used to improve human health and to treat various ailments such as kidney problems, bladder inflammation, gout, diabetes and cancer. This study sought to prepare the $O$. staminues ethanolic extract in PVP/Poloxamer 407 solid dispersions in order to improve the aqueous solubility of the extract's major components including rosmarinic acid, TMF, eupatorin and sinensetin. Solid dispersions were prepared by the solvent evaporation method, and the resulting formulation was studied by HPLC, FTIR, TEM, particle size and zeta potential. Drug release through dialysis membrane was also included. HPLC analysis showed significant improvement in aqueous solubility of the marker compounds compared to the unformulated extract; FTIR analysis did not indicate the existence of an interaction between the extract and the carrier; TEM analysis of the reconstituted formulation showed the presence of rounded nanoparticles; the particle size analysis indicates the presence of anionic particles with hydrodynamic diameter of $<200 \mathrm{~nm}$. The drug release studies showed $>90 \%$ release of the unformulated extract compared to $<40 \%$ in the formulated extract. Our results show improvement in solubility of the $O$. staminues ethanolic extract, however with slow release pattern. This can be explained due to particle size reduction and entrapment of the extract components within the core of the particles formed by the carriers. In conclusion, the PVP/Poloxamer 407 solid dispersions of the $O$. staminues ethanolic extract may provide a sustained release formulation which may improve the overall therapeutic value of this medicinal herb.

Keywords: Orthosiphon stamineus, solid dispersion, solubility enhancement. 